(2) Open Access Full Text Article

\title{
Hypofractionated stereotactic body radiation therapy for elderly patients with stage IIB-IV nonsmall cell lung cancer who are ineligible for or refuse other treatment modalities
}

\author{
This article was published in the following Dove Press journal: \\ Lung Cancer: Targets and Therapy \\ 3 October 2014 \\ Number of times this article has been viewed
}

\section{Sana D Karam' \\ Zachary D Horne ${ }^{2}$ \\ Robert L Hong ${ }^{2}$ \\ Don McRae ${ }^{2}$ \\ David Duhamel ${ }^{3}$ \\ Nadim M Nasr ${ }^{2}$ \\ 'Department of Radiation Oncology, University of Colorado, Denver, CO, USA; ${ }^{2}$ Department of Radiation Oncology, ${ }^{3}$ Department of Pulmonary/ Critical Care Medicine, Virginia Hospital Center, Arlington, VA, USA}

Correspondence: Nadim M Nasr Department of Radiation Oncology, Virginia Hospital Center I70I N George Mason Dr, Arlington, VA 22205, USA

Emailnmnasr@gmail.com
Objective: In elderly patients with stage IIB-IV nonsmall cell lung cancer who cannot tolerate chemotherapy, conventionally fractionated radiotherapy is the treatment of choice. We present our experience with hypofractionated stereotactic body radiation therapy (SBRT) in the treatment of this patient population.

Methods: Thirty-three patients with a median age of 80 years treated with fractionated SBRT were retrospectively analyzed. Most patients were smokers and had preexisting lung disease and either refused treatment or were ineligible. A median prescribed dose of 40 Gy was delivered to the prescription isodose line over a median of five treatments. The majority of patients $(70 \%)$ did not receive chemotherapy.

Results: With a median follow-up of 9 months (range: 4-40 months), the actuarial median overall survival (OS) and progression-free survival were 12 months for both. One year actuarial survival outcomes were $75 \%, 58 \%, 44 \%$, and $48 \%$ for local control, regional control, progressionfree survival, and OS, respectively. Increased volume of disease was a statistically significant predictor of worse OS. Three patients developed a grade 1 cough that peaked 3 weeks after treatment and resolved within 1 month. One patient developed grade 1 tracheal mucositis and three patients developed grade 1 pneumonitis. Both resolved 6 weeks after treatment. Three patients died within the first month of treatment, but the cause of death did not appear to be related to the treatment

Conclusion: Hypofractionated SBRT is a relatively safe and convenient treatment option for elderly patients with inoperable stage IIB-IV nonsmall cell lung cancer. However, given the small sample size and the heterogeneity of the patient population, larger studies are needed before adopting this treatment option into clinical practice.

Keywords: stereotactic body radiation therapy, CyberKnife, stage IIB, stage IIIA, stage IV, nodal, chemotherapy

\section{Introduction}

Radiotherapy, alone or combined with chemotherapy remains the most commonly used modality for the treatment of locally advanced (LA) nonsmall cell lung cancer (NSCLC). ${ }^{1}$ In the positron emission tomography (PET) staging era, concurrent chemoradiation (cCRT) has become the standard of care in treating LA NSCLC with median survival rates of approximately 21 months. ${ }^{1}$ However, many patients are not suitable for this treatment based on poor performance status and comorbidities. Sequential chemoradiation (CRT) is an alternative modality, although a meta-analysis of cCRT 
versus sequential CRT has shown that concurrent treatment results in decreased locoregional progression and an absolute overall survival (OS) benefit of $5.7 \%$ at 3 years and $4.5 \%$ at 5 years. ${ }^{2}$ In elderly patients and those with comorbidities, data are lacking as these patients are generally deemed ineligible for inclusion in clinical trials with cCRT. ${ }^{3}$ Elderly patients, who constitute approximately two-thirds of NSCLC cases, ${ }^{4}$ generally have decreased tolerance to aggressive cancer treatments and an increased incidence of toxic effects. ${ }^{3}$ The convenience of administering the treatment over a course of 5 days is also very appealing to those who perceive the prolonged treatment course of the conventional approach as a major challenge to treatment delivery.

Hypofractionated radiotherapy (RT) has been attempted as a means to substantially shorten the course of treatment, thus providing rapid symptom relief, convenience to patients, and opportunities for efficient resource utilization. ${ }^{5-7}$ Results from earlier trials, however, have been conflicting on whether it is an improvement over standard palliative fractionation. ${ }^{5}$ In a study aimed at examining the experience with hypofractionated RT for the palliation of stage III and IV NSCLC, severe adverse effects were shown by a large proportion of cases involving the skin, soft tissues of the chest wall, and lungs $(55.5 \%) .{ }^{5}$ However, a landmark study that compared three different fractionation regimens showed equivalent survival outcomes for stage IIIB NSCLC without an increase in adverse toxicity. ${ }^{6}$

Recent advances in radiation delivery techniques, which provide highly conformal dose distributions, have yielded better tolerance as they have been more radiobiologically forgiving with respect to hypofractionation. ${ }^{7}$ A new generation of machines including the CyberKnife ${ }^{\circledR}$ (CK) system allows for accurate dose delivery to the planned target volume (PTV) while avoiding the organs at risk. ${ }^{8}$ The goal of this study is to retrospectively review the safety profile of using hypofractionated CK-based radiation therapy for medically inoperable elderly patients with stage IIB-IV lung cancer who are otherwise ineligible for conventional treatment.

\section{Methods}

\section{Eligibility}

After research ethics board approval was obtained, all patients with a diagnosis of clinically staged IIB-IV NSCLC (per the American Joint Committee on Cancer staging manual, 7th edition) treated between 2009 and 2012 with either definitive or palliative intent hypofractionated RT at the Virginia Hospital Center were identified. All patients had a histologically confirmed diagnosis of NSCLC, and all had available a medical history; a physical examination; computed tomography (CT) imaging of the chest, abdomen, and pelvis; PET scan and brain magnetic resonance imaging (MRI)/ CT. All patients were determined by thoracic surgeons to be medically inoperable. Exclusion criteria included recurrent disease, previous irradiation, or a history of surgery. Charts were reviewed to determine patterns of disease failure, toxicity (as defined by the Common Terminology Criteria for Adverse Events, version 4.3), and outcomes.

\section{Treatment planning}

Most patients (75\%) received fiducial placement for real-time image guidance, as previously described. ${ }^{8}$ Fiducial placement was done as we have previously described. ${ }^{9}$ For lesions that were adjacent to, and did not move independent of the spine $(25 \%)$, spinal tracking that uses spine bony landmarks (X-Sight ${ }^{\mathrm{TM}}$ ) was utilized instead. ${ }^{8}$ Fine-cut $(1.25 \mathrm{~mm}) \mathrm{CT}$ with intravenous contrast was used for targeting and treatment planning. The gross tumor volume (GTV) was generally drawn using CT pulmonary windows. However, a soft tissue window with contrast was sometimes used to avoid inclusion of adjacent vessels, atelectasis, or mediastinal or chest wall structures within the GTV.PET/ CT fusion was done on all patients, and PET data were used in defining the GTV. An additional margin of $5 \mathrm{~mm}$ was added to the GTV to constitute the PTV, but adjustments were made by the treating physician based on tumor location, proximity of critical structures, and tumor motion during treatment. For patients treated with palliative intent, only tumors within the chest were targeted for radiation planning. The following critical structures were contoured: spinal cord; esophagus; brachial plexus; heart; trachea and proximal bronchial tree; proximal trachea; whole lung; and skin. As a general rule, prescription doses were dictated by the tolerance of surrounding structures, which were in accordance with the American Association of Physicists in Medicine (AAPM) Task Group 101. ${ }^{10}$

\section{Radiosurgery dosimetry}

Three-dimensional noncoplanar beam arrangements were custom designed for each case to deliver highly conformal prescription dose distributions. Treatment conformity was determined in all patients using the new conformity index, which was calculated by the following formula: ${ }^{2}$

Treatment volume $\times$ prescription isodose line /(volume of target covered by prescription isodose line).

Generally, more beams were used for larger lesions. As such, prescription lines covering the PTV were typically 
around $80 \%$ but they ranged between the $60 \%-90 \%$ line rather than the more traditional $95 \%-100 \%$ line. Treatment homogeneity was sacrificed for conformality, and higher isodoses were manipulated to occur within the target and not in adjacent normal tissue. Up to $20 \%$ of treatment inhomogeneities were accepted within the tumors, especially for larger lesions. In other words, risk adaptive RT was used, whereby the selective boosting of tumor volumes was accomplished without violating normal tissue complication constraints using information from functional imaging. Biologically effective dose (BED) was calculated using the following formula:

$$
\mathrm{BED}=\mathrm{nd}(1+\mathrm{d} / \alpha / \beta)
$$

where $\mathrm{n}$ is the number of fractions and $\mathrm{d}$ is the dose per fraction, and using an $\alpha / \beta$ ratio of 10 for acute reacting tissues such as lung.

\section{Follow-up and statistical analysis}

Clinical examinations and PET/CT imaging were performed at 3-month follow-up intervals from the end of treatment. Progression-free survival (PFS) was defined as the time from the first day of stereotactic body radiation therapy (SBRT) treatment to local, regional, or distant failure or to the last follow-up in living patients without evidence of recurrence or progression. Local control (LC), local failure, regional failure, and distant failures were defined as previously described. ${ }^{11}$ Patients were censored at the time of death. OS was the time from SBRT treatment until death or the last follow-up. Interpretation of available fludeoxyglucose (FDG)-PET/CT, and CT scans with correlative clinical examinations were used to assess the response of the treated lesion 3 months after SBRT. All of our follow-up was reviewed in an interdisciplinary tumor board format in the presence of board certified radiologist and a nuclear medicine physician and a consensus agreement for clinical decision making. In general, the PET interpretation by our nuclear medicine physicians closely followed what had been reported previously, ${ }^{12}$ whereas the CT definition of locoregional failure mirrored published criteria. ${ }^{13}$

Log-rank tests and Cox regression models were used to evaluate the association between clinical factors and each survival outcome. The independent variables considered were stage (IIB, IIIA, IV), intent (curative, palliative), metastatic status (yes, no), sex (male, female), BED ( $<100 \mathrm{~Gy}$, $>100$ Gy), chemotherapy (yes, no), nodal status $(<\mathrm{N} 2$, $\geq \mathrm{N} 2$ ), age in years, GTV in cubic centimeters (cc), SBRT dose in Gy, smoking in number of pack years, and Karnofsky performance status. Kaplan-Meier plots are presented for selected significant factors. Acute toxicities examined included fatigue, chest pain, shortness of breath, cough, hemoptysis, wheezing, and esophagitis that occurred during treatment or within the first 2 weeks following the end of treatment. Radiation pneumonitis was examined as long-term toxicity in all patients. Analyses were performed in SAS version 9.2 (SAS Institute Inc., Cary, NC, USA).

\section{Results \\ Patient characteristics}

A total of 81 patients and 99 lesions with stage IIB-IV NSCLC were treated at our institution. Patients with recurrent tumors who were reirradiated and those who received hypofractionated SBRT as a means of dose escalation were excluded from the analysis, leaving a total of 38 patients. Five patients were further excluded from the analysis. Two patients did not have a biopsy proven tumor, two patients had no follow-up information, and one had no PET CT. A total of 33 patients with stage IIB-IV NSCLC were therefore included in this study. Baseline patient and disease characteristics are listed in Table 1. Median patient age at the time of treatment was 79 years (range: 65-100 years) for all patients, with $24 \%$ males and $76 \%$ females. The majority ( $88 \%$ ) of the patients were smokers, with a median Karnofsky performance status of 70 , and $85 \%$ had a diagnosis of chronic obstructive pulmonary disease (COPD). The histology varied between squamous (51\%), adenocarcinoma (33\%), and nonspecified NSCLC (15\%). Forty-two percent of patients had metastatic disease, while $33 \%$ had stage IIIA, and $24 \%$ had stage IIB disease. Fifty-eight percent of the patients were treated with

Table I Patient characteristics

\begin{tabular}{ll}
\hline Age (median; range) & $(79$ years; \\
& $65-100$ years) \\
KPS (median; range) & $(70 ; 50-100)$ \\
Sex (male; female) & $(24 \% ; 76 \%)$ \\
Smoker (yes; no) (median; range) & $(88 \% ; 12 \%)$ \\
& $(50 ; 0 \%-110 \%)$ \\
Histology (adenocarcinoma; squamous; NSCLC) & $(33 \% ; 51 \% ; 15 \%)$ \\
Clinical stage (IIB; IIIA; IV) & $(24 \% ; 33 \% ; 42 \%)$ \\
N stage (0; I; 2; 3) & $(51.5 \% ; 9.1 \% ;$ \\
& $36.4 \% ; 3.0 \%)$ \\
Metastatic disease (M0; MIa; MIb) & $(58 \% ; 15 \% ; 27 \%)$ \\
Maximum diameter in cm (mean; median; range) & $(3.9 ; 3.6 ; 1.1-7.2)$ \\
Volume in cc (mean; median; range) & $(88.1 ; 88.8 ; 76-220)$ \\
Tumor location (upper; lower; right; left; & $(74 \% ; 26 \% ; 58 \% ;$ \\
paratracheal lymph node) & $36 \% ; 6 \%)$ \\
Tumor location (central; peripheral) & $(42 \% ; 58 \%)$ \\
\hline
\end{tabular}

Abbreviations: KPS, Karnofsky performance status; NSCLC, nonsmall cell lung cancer. 
curative intent and all had M0 disease. Forty-two percent were treated palliatively and none of the patients with metastatic disease were treated with a curative intent. However, $58 \%$ of the patients without metastatic disease (M0 patients) were treated with palliative intent. The majority ( $51 \%$ ) of all patients treated had N0 disease with a median tumor volume of $88.1 \mathrm{cc}$. Most of the tumors were located peripherally in the right upper lobe (Table 1).

For all participants, $42 \%$ had metastatic disease, while $33 \%$ had stage IIIA, and $24 \%$ had stage IIB disease. For the $58 \%$ (19) of the patients who were treated with curative intent, $42 \%$ were stage IIB and 58\% were stage IIIA. While the majority (51\%) of all patients treated had N0 disease, most of those treated with curative intent had N2 disease (48\%). The median tumor volume was $88.1 \mathrm{cc}$ for all tumors and $108.5 \mathrm{cc}$ for those treated with curative intent. Most of the tumors were located peripherally in the right upper lobe (Table 1).

\section{Treatment characteristics}

A median prescribed dose of 40 Gy was delivered to the prescription isodose line over a median of four 1-2 hour treatments over 5-10 days (median: 7 days). The median BED10 was 77.8 Gy (range: 42.62-132 Gy). On average (mean and median), approximately $95 \%$ of the target volume (PTV) was conformally covered by the prescription isodose line (range: $75 \%-99.85 \%$ ). The median conformity index was 1.50 (range: 1.19-2.52). The median percent of the total lung volume receiving 15 Gy or more was $6.5 \%$. The majority of patients (70\%) did not receive chemotherapy. For patients who received chemotherapy or targeted agents, a minimum of 2 weeks was allowed prior to the initiation of radiation therapy in relation to chemotherapy administered either adjuvantly or neoadjuvantly. For bevacizumab-based chemotherapy, a minimum of 1 month was required on either end of treatment.

\section{Clinical outcomes and prognostic factors}

The median follow-up was 9 months (range 0-40 months) with a median OS of 12 months and a median PFS of 12 months. The median time LC was not reached. The actuarial 1-year OS, PFS, LC, regional control (RC), and distant control rates were, $48 \%, 44 \%, 75 \%, 58 \%$, and $70 \%$, respectively (Figure 1). The pattern of failure differed by treatment intent. For those treated with curative intent, the predominant pattern of failure was locoregional with actuarial local and RC rates of $64 \%$. For those treated palliatively, the failure rates were evenly distributed between regional and distant, with actuarial $\mathrm{RC}$ and distant control rates of $68 \%$. Univariate analysis showed treatment volume to be a statistically significant predictor of OS, with those below the median level of 88.8 cc predictive for improved OS (hazard ratio $=3.03$; $95 \%$ confidence interval $=1.23-7.81 ; P=0.02$ ) (Figure 2 ). While our analysis shows stage of disease to be predictive of OS, a comparison of all stages showed that stage IIB had superior outcomes compared to stage IV, but even worse survival outcomes for stage IIIA $(P=0.05)$. For stages IIB, IIIA, IV, the respective actuarial 1-year OS rates were $40 \%$, $60 \%$, and $85 \%$ with a corresponding median OS of 9 months, 14 months, and 21 months. The difference between the groups, however, disappears on multivariate analysis after controlling for volume of disease.

\section{Toxicity}

Since the majority of the patients (85\%) had COPD at baseline, toxicity was focused on evaluating new problems or exacerbation of their baseline problems. Three patients developed grade 1 cough. Generally, the cough surfaced during the week of treatment, peaked within 3 weeks, and resolved within 4 months after treatment. One patient developed tracheal mucositis from fiducial placement during bronchoscopy that resolved prior to starting treatment. Another patient developed grade 1 tracheal mucositis 2 weeks following treatment, but it had resolved by the 3-month follow-up. Three patients developed grade 1 pneumonitis. This was reported on their 4-week follow-up and was resolved by their 6-week follow-up. There were two other cases of pneumonia reported, but it was not clear whether the etiology was related to radiation treatment. One patient was admitted with bacterial pneumonia 2 months following treatment. Another patient was admitted with a diagnosis of Candida albicans 9 months following treatment. Only one patient developed grade 2 dyspnea appearing toward the end of her treatment, with a peak at about 1 month post-treatment and resolving by the 4-month follow-up visit. One patient developed grade 1 new onset pleuritic pain that resolved by the 3-month follow-up. Three patients died approximately within the first month of treatment. The cause of death was uncertain for all three. One patient was an 82-year-old female who was being treated for a T3N2M0 tumor for squamous cell carcinoma of the right upper lobe with poor performance status; this patient was deemed ineligible for chemotherapy. She had reported chest pain while on treatment that self-resolved. She was eventually discharged to a hospice and died 42 days posttreatment. The two other patients were treated palliatively and had an established diagnosis of metastatic disease. 

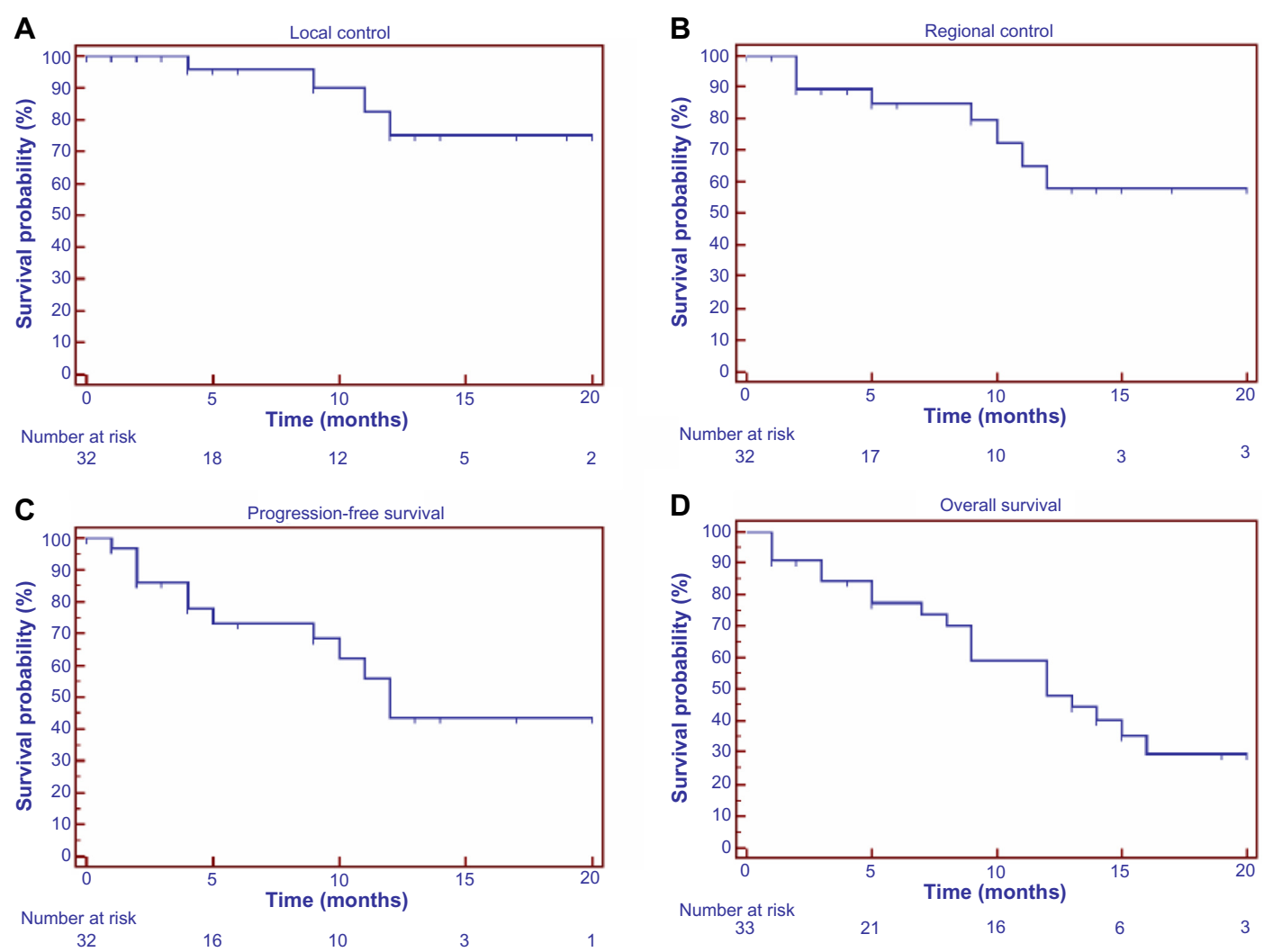

Figure I Survival outcomes of all patients with early-stage lung cancer (number $=33$ ).

Notes: (A) Local control; (B) regional control; (C) progression-free survival; (D) overall survival.

\section{Discussion}

The standard treatment for LA lung cancer is cCRT. ${ }^{14,15}$ However, many patients cannot tolerate the regimen because of its toxicity. ${ }^{3}$ Sequential chemotherapy followed by RT is used in these situations with good outcomes. ${ }^{16}$ For patients who cannot tolerate the use of any chemotherapy because of comorbid conditions, radiation therapy alone is used. ${ }^{1,3}$ Hypofractionated regimens consist of fewer fractions with higher doses per fraction. ${ }^{7}$ Not only do large fractions offer the added convenience of shortened treatment course for

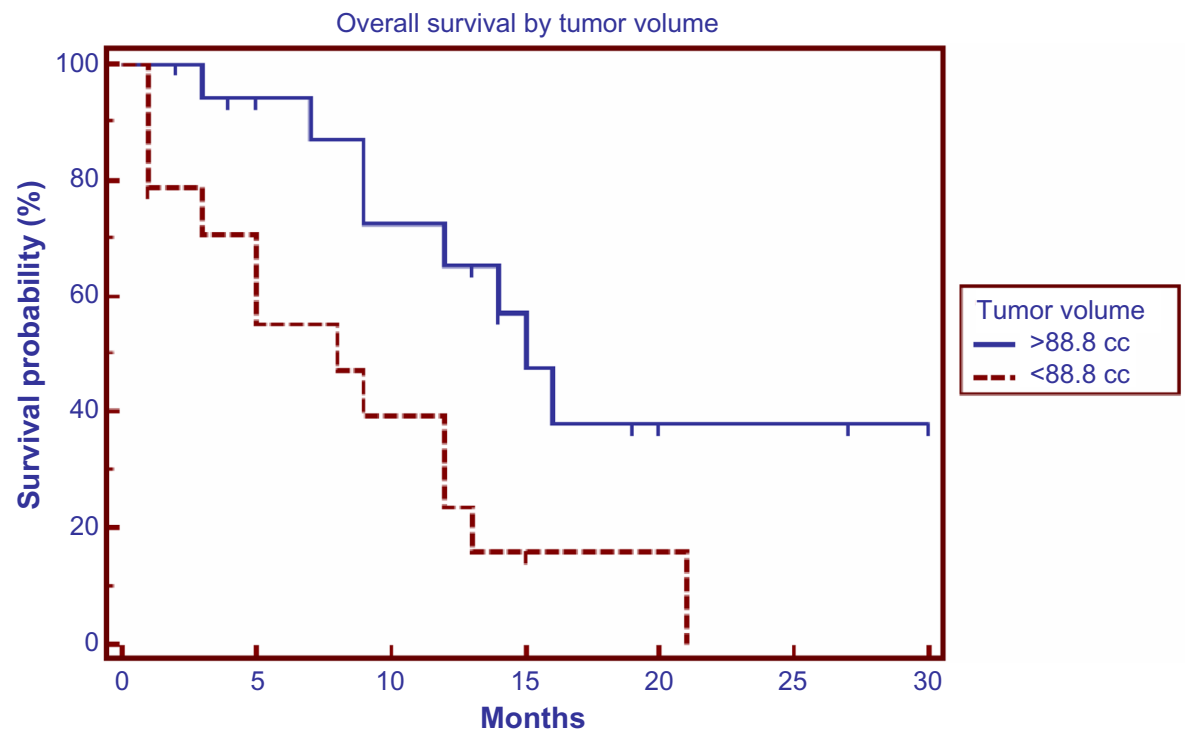

Figure $\mathbf{2}$ Overall survival for all patients by volume. 
elderly and/or debilitated patients, but large fractions may improve disease control by obtaining a higher biological effectiveness. The potential tumor doubling time, an indicator of the proliferation rate, has been found to have a median value of 7 days for NSCLC and has been shown to have prognostic significance for this disease. ${ }^{17,18}$ Employing a shortened regimen in the case of rapidly repopulating tumors, which can be achieved with hypofractionation, would therefore be beneficial. ${ }^{17}$ Hypofractionated radiation therapy is also based on the concept that the accelerated repopulation of tumor cells that increases later in the course of treatment could diminish the potential benefits of dose escalation in standard fractionation. ${ }^{1,19,20}$ A recent review of published clinical data for NSCLC patients treated with standard RT, continuous hyperfractionated accelerated radiation therapy, or hypofractionated regimens found best outcomes for hypofractionated RT (overall treatment time of $<6$ weeks) in terms of 2-year disease-free survival compared to continuous hyperfractioned accelerated radiation therapy or prolonged conventionally fractionated treatments, converting the prescribed dose to $\mathrm{BED}$, with a correction for repopulation. ${ }^{21}$ No clear correlation was observed between the dose delivered to the tumor and toxicity rates (pneumonitis/esophagitis). ${ }^{21}$ Also, low toxicity rates were reported for hypofractionated RT, which means that dose escalation can be conducted safely. ${ }^{21}$

Multiple retrospective analyses using hypofractionated RT for stage IIB-IV NSCLC have been published. Slotman et $\mathrm{l}^{6}$ retrospectively compared three hypofractionated schemes for the treatment of unresectable NSCLC (stage IIIA-IV) (40 Gy split course; 30-32 Gy in six fractions, or 24 Gy in three fractions) and demonstrated that a split-course treatment regimen of 40 Gy had improved OS and lowered local relapse rates in stage IIIA NSCLC patients, but not in patients with stage IIIB-IV disease. Kepka et $\mathrm{al}^{22}$ performed a dose escalation study in which patients were initially treated with a 4-week course (21 days) at 56.7 Gy (2.7 Gy per fraction), and gradually escalated to a mean dose of 60.9 Gy in 21 days (2.9 Gy per fraction). Fifty patients received induction chemotherapy for $2-3$ cycles. The median survival was 17 months, and the 2- and 3-year OS rates were $32 \%$ and $19 \%$, respectively. ${ }^{22}$ A recently published analysis of a large retrospective series comparing two standard fractionation regimens to an accelerated hypofractionated regimen given in 45 Gy over 3 weeks showed tolerable toxicity and no difference in survival outcomes between the groups after adjusting for confounding variables. ${ }^{23}$

Currently, only a few studies have investigated the efficacy and tolerance of hypofractionated radiation schedules in LA NSCLC. These studies demonstrated acceptable LC and toxicity rates after hypofractionated RT based on a three-dimensional conformal radiation therapy planning system. ${ }^{24-26}$ Patients in these trials were younger patients with better performance status, and a significantly larger percentage of patients were able to receive chemotherapy. In another study that focused on elderly patients but included both stage I and stage II disease, hypofractionated RT resulted in good survival outcomes and tolerable toxicity. ${ }^{27}$

The adoption of a smaller irradiated volume in hypofractionated treatment for stage IIB-IV NSCLC is accomplished by greater setup precision. SBRT with real-time fiducial tracking combined with the use of FDG-PET/CT for the identification of nodal volumes and treatment-resistant areas in the tumor allow for high precision delivery of hypofractionated therapy for LA lung cancer. ${ }^{28}$ It is important to emphasize the importance of PET in this setting as SBRT to PET-positive tumor areas increases RT dose for locoregional control because of the high biologically equivalent dose achieved with SBRT without increasing toxicity. Since the lung is considered a parallel organs at risk, high dose to small areas are possible as tissue can tolerate loss to part of their structure and continue to function. ${ }^{17}$ Hypofractionated SBRT allows for patient-specific margins and a reduction in the exposure of normal tissue to radiation, leading to the treatment of patients who were previously not suitable for radical treatment and potential dose escalation.

The hypofractionated SBRT system with real-time fiducial tracking in patients with LA NSCLC has been reported in a previous publication. Lv et $\mathrm{al}^{29}$ retrospectively analyzed survival outcomes of 34 histologically proven NSCLC tumors that were treated with $\mathrm{CK}$ with a total dose of 35-60 Gy given in 2-5 fractions. At an unspecified median follow-up period, the response rate and disease control rates were reportedly $58 \%$ and $81 \%$, respectively, with negligible toxicity. The majority of their patients were younger and had received chemotherapy. ${ }^{29}$ Even in elderly patients with LA NSCLC, the addition of chemotherapy given sequentially has been shown to be superior to RT alone in a recent Phase III randomized clinical trial. ${ }^{16,30}$ Nevertheless, our results are comparable. The 1-year PFS for all patients in our cohort was 44\% with a median time to progression of 12 months. The 1-year LC rate was $75 \%$, and none of those with metastatic disease failed locally. Tumor volume was considerably smaller in the palliative intent group and the majority of those patients had also received chemotherapy, both of which could bias the LC data. On univariate analysis, tumor volume over the median value of $88.8 \mathrm{cc}$ was predictive of poor OS. 


\section{Conclusion}

The majority of patients with LA NSCLC, particularly elderly patients with multiple comorbid medical conditions, are not suitable for the gold standard treatment of cCRT; therefore, novel strategies integrating RT technological advances and radiobiological knowledge need to be evaluated. Hypofractionated SBRT allows individualized radiation dose escalation based on normal tissue constraints and overall shorter treatment time. Our results show promise for using hypofractionated CK-based SBRT for elderly patients who are ineligible for standard treatment, or for those with multiple comorbid medical conditions. Given the retrospective nature of the analysis, the small sample size, and the heterogeneity of the patient population, the conclusions can only be limited by the safety of the application of this procedure. Even then, toxicity data are limited by the short and sometimes poor follow-up. This is particularly relevant as toxicities from SBRT are usually of late onset. Additionally, the lack of clear cutoff values post-treatment SUVpeak (or SUVmax) that could be identified for use in clinical decision making or follow-up ${ }^{31}$ underscore the limitation of using PET as a biomarker for treatment response. Until there are clear guidelines that dictate what SUVmax cutoff values to use in clinical practice, the conclusions should be interpreted with caution, and future studies with increased sample size, longer follow-up, and a less heterogeneous population are warranted before routinely incorporating this treatment modality into clinical practice.

\section{Acknowledgment}

Institutional Review Board exemption from a full board review was obtained prior to research commencement.

\section{Disclosure}

The authors report no conflicts of interest in this work.

\section{References}

1. McCloskey P, Balduyck B, Van Schil PE, Faivre-Finn C, O’Brien M. Radical treatment of non-small cell lung cancer during the last 5 years. Eur J Cancer. 2013;49(7):1555-1564.

2. Aupérin A, Le Péchoux C, Rolland E, et al. Meta-analysis of concomitant versus sequential radiochemotherapy in locally advanced non-small-cell lung cancer. J Clin Oncol. 2010;28(13):2181-2190.

3. Bayman N, Alam N, Faivre-Finn C. Radiotherapy for lung cancer in the elderly. Lung Cancer. 2010;68(2):129-136.

4. Jemal A, Thun MJ, Ries LA, et al. Annual report to the nation on the status of cancer, 1975-2005, featuring trends in lung cancer, tobacco use, and tobacco control. J Natl Cancer Inst. 2008;100(23):1672-1694.

5. Pirtoli L, Bindi M, Bellezza A, Pepi F, Tucci E. Unfavorable experience with hypofractionated radiotherapy in unresectable lung cancer. Tumori. 1992;78(5):305-310.
6. Slotman BJ, Njo KH, de Jonge A, Meijer OW, Karim AB. Hypofractionated radiation therapy in unresectable stage III non-small cell lung cancer. Cancer. 1993;72(6):1885-1893.

7. Vogelius IS, Westerly DC, Cannon GM, Bentzen SM. Hypofractionation does not increase radiation pneumonitis risk with modern conformal radiation delivery techniques. Acta Oncol. 2010;49(7):1052-1057.

8. Gibbs IC, Loo BW Jr. CyberKnife stereotactic ablative radiotherapy for lung tumors. Technol Cancer Res Treat. 2010;9(6):589-596.

9. Karam SD, Horne ZD, Hong RL, McRae D, Duhamel D, Nasr NM. Dose escalation with stereotactic body radiation therapy boost for locally advanced non small cell lung cancer. Radiat Oncol. 2013;8:179.

10. Benedict SH, Yenice KM, Followill D, et al. Stereotactic body radiation therapy: the report of AAPM Task Group 101. Med Phys. 2010;37(8): 4078-4101.

11. Timmerman RD. Surgery versus stereotactic body radiation therapy for early-stage lung cancer: who's down for the count? J Clin Oncol. 2010;28(6):907-909.

12. MacManus M, Nestle U, Rosenzweig KE, et al. Use of PET and PET/ CT for radiation therapy planning: IAEA expert report 2006-2007. Radiother Oncol. 2009;91(1):85-94.

13. Machtay M, Paulus R, Moughan J, et al. Defining local-regional control and its importance in locally advanced non-small cell lung carcinoma. J Thorac Oncol. 2012;7(4):716-722.

14. Le Chevalier T, Arriagada R, Quoix E, et al. Radiotherapy alone versus combined chemotherapy and radiotherapy in nonresectable non-smallcell lung cancer: first analysis of a randomized trial in 353 patients. J Natl Cancer Inst. 1991;83(6):417-423.

15. Kim TY, Yang SH, Lee SH, et al. A phase III randomized trial of combined chemoradiotherapy versus radiotherapy alone in locally advanced non-small-cell lung cancer. Am J Clin Oncol. 2002;25(3):238-243.

16. Atagi S, Kawahara M, Yokoyama A, et al; Japan Clinical Oncology Group Lung Cancer Study Group. Thoracic radiotherapy with or without daily low-dose carboplatin in elderly patients with non-small-cell lung cancer: a randomised, controlled, phase 3 trial by the Japan Clinical Oncology Group (JCOG0301). Lancet Oncol. 2012;13(7):671-678.

17. Abratt RP, Bogart JA, Hunter A. Hypofractionated irradiation for nonsmall cell lung cancer. Lung Cancer. 2002;36(3):225-233.

18. Tinnemans MM, Lenders MH, ten Velde GP, Blijham GH Ramaekers FC, Schutte B. Prognostic value of cytokinetic parameters in lung cancer after in vivo bromodeoxyuridine labelling. Anticancer Res. 1999;19(1A):531-534.

19. Withers HR, Taylor JM, Maciejewski B. The hazard of accelerated tumor clonogen repopulation during radiotherapy. Acta Oncol. 1988;27(2):131-146.

20. van Baardwijk A, Bosmans G, Bentzen SM, et al. Radiation dose prescription for non-small-cell lung cancer according to normal tissue dose constraints: an in silico clinical trial. Int J Radiat Oncol Biol Phys. 2008;71(4):1103-1110.

21. Partridge M, Ramos M, Sardaro A, Brada M. Dose escalation for nonsmall cell lung cancer: analysis and modelling of published literature. Radiother Oncol. 2011;99(1):6-11.

22. Kepka L, Tyc-Szczepaniak D, Bujko K. Dose-per-fraction escalation of accelerated hypofractionated three-dimensional conformal radiotherapy in locally advanced non-small cell lung cancer. JThorac Oncol. 2009;4(7):853-861.

23. Amini A, Lin SH, Wei C, Allen P, Cox JD, Komaki R. Accelerated hypofractionated radiation therapy compared to conventionally fractionated radiation therapy for the treatment of inoperable non-small cell lung cancer. Radiat Oncol. 2012;7:33.

24. Zhu ZF, Fan M, Wu KL, et al. A phase II trial of accelerated hypofractionated three-dimensional conformal radiation therapy in locally advanced non-small cell lung cancer. Radiother Oncol. 2011;98(3):304-308.

25. Osti MF, Agolli L, Valeriani M, et al. Image guided hypofractionated 3-dimensional radiation therapy in patients with inoperable advanced stage non-small cell lung cancer. Int J Radiat Oncol Biol Phys. 2013;85(3):e157-e163. 
26. Salazar OM, Sandhu TS, Lattin PB, et al. Once-weekly, high-dose stereotactic body radiotherapy for lung cancer: 6-year analysis of 60 early-stage, 42 locally advanced, and 7 metastatic lung cancers. Int J Radiat Oncol Biol Phys. 2008;72(3):707-715.

27. Bonfili P, Di Staso M, Gravina GL, et al. Hypofractioned radical radiotherapy in elderly patients with medically inoperable stage I-II non-small-cell lung cancer. Lung Cancer. 2010;67(1):81-85.

28. Nagata Y, Matsuo Y, Takayama K, et al. Current status of stereotactic body radiotherapy for lung cancer. Int J Clin Oncol. 2007;12(1):3-7.

29. Lv Y, Wang Z, Zhu X, et al. [Preliminary effect of Cyberknife radiosurgery in the treatment of 31 patients with advanced non-small cell lung cancer]. Zhongguo Fei Ai Za Zhi. 2011;14(4):329-334. Chinese.
30. Stinchcombe TE, Bogart JA. Novel approaches of chemoradiotherapy in unresectable stage IIIA and stage IIIB non-small cell lung cancer. Oncologist. 2012;17(5):682-693.

31. Machtay M, Duan F, Siegel BA, et al. Prediction of survival by [18F] fluorodeoxyglucose positron emission tomography in patients with locally advanced non-small-cell lung cancer undergoing definitive chemoradiation therapy: results of the ACRIN 6668/RTOG 0235 trial. J Clin Oncol. 2013;31(30):3823-3830.

\section{Publish your work in this journal}

Lung Cancer: Targets and Therapy is an international, peer-reviewed, open access journal focusing on lung cancer research, identification of therapeutic targets and the optimal use of preventative and integrated treatment interventions to achieve improved outcomes, enhanced survival and quality of life for the cancer patient. Specific topics covered in the journal include: Epidemiology, detection and screening; Cellular research and biomarkers; Identification of biotargets and agents with novel

mechanisms of action; Optimal clinical use of existing anticancer agents, including combination therapies; Radiation and surgery; Palliative care; Patient adherence, quality of life, satisfaction; Health economic evaluations. The manuscript management system is completely online and includes a very quick and fair peer-review ystem. Visit http:/WWw.dovepress.com/testimonils.php to read real quotes from

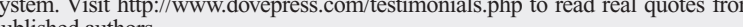
published authors. 